\title{
Erratum to: Per-Session Security: Password-Based Cryptography Revisited
}

Grégory Demay, Peter Gaži, Ueli Maurer, and

Björn Tackmann

\section{Erratum to: \\ Chapter "Per-Session Security: Password-Based \\ Cryptography Revisited" in: S.N. Foley et al. (Eds.): \\ Computer Security - ESORICS 2017, Part I, LNCS 10492, https://doi.org/10.1007/978-3-319-66402-6_24}

The footnote at the end of the title page has been corrected by the authors. Correctly it reads:

G. Demay-Work done while author was at ETH Zürich and supported by the Zurich Information Security and Privacy Center.

P. Gaži-Work done while author was at ETH Zürich and IST Austria, in part supported by the ERC grants 259668-PSPC and 682815-TOCNeT.

B. Tackmann-Work done while author was at ETH Zürich and UC San Diego, in part supported by SNF fellowship P2EZP2-155566 and NSF grant CNS-1228890.

The full version is available at https://eprint.iacr.org/2016/166.

The updated online version of this chapter can be found at https://doi.org/10.1007/978-3-319-66402-6_24 